\title{
LA FORMACIÓN DE MAESTROS Y MAESTRAS ELEMENTALES EN ESPAÑA A FINALES DEL SIGLO XIX. EL CASO DE LA GEOMETRÍA
}

\author{
Vicente Meavilla Seguí \\ Antonio Oller Marcén \\ Universidad de Zaragoza
}

\begin{abstract}
RESUMEN. Durante el siglo XIX en España, la discriminación por género en la educación de niños y niñas fue una práctica que se extendió a la formación de los futuros maestros y maestras de primera enseñanza. En este artículo analizamos dichas diferencias a partir del estudio de la legislación de la época. Posteriormente nos centramos en el caso de la Geometría. En concreto, analizamos dos manuales de preparación de opositores y concluimos que las diferencias entre la formación de maestros y maestras no se daban a nivel matemático, sino en cuanto a las aplicaciones y la formación didáctica. No obstante, dichos manuales contribuían a perpetuar el papel pasivo y secundario de la mujer a través de comentarios de carácter filosófico.
\end{abstract}

PALABRAS CLAVE: Geometría, siglo XIX, primera enseñanza, magisterio, discriminación por género.

\section{ELEMENTARY MALE AND FEMALE TEACHER TRAINING IN SPAIN AT THE ENDING OF XIX CENTURY. THE CASE OF GEOMETRY}

ABSTRACT: During XIX century in Spain, gender discrimination of boys and girls was a practice that extended to the training of prospective teachers of elementary education. In this paper, we analyze these differences studying the legislation at that time. Then we focus on the case of Geometry. In particular, we analyze two textbooks addressed to prospective teachers and we conclude that there were no differences at a mathematical level, but regarding applications and pedagogical training. Nevertheless, those textbooks contributed to perpetuate the passive and secondary role of women by means of philosophical commentaries.

KEYWORDS: Geometry, XIX century, elementary education, school teaching, gender discrimination. 
Correspondencia: Vicente Mediavilla Seguí, Universidad de Zaragoza, Departamento de Matemáticas, Edificio de Matemáticas, 50009 Zaragoza. Email: vmeavill@hotmail.com.

\section{INTRODUCCIÓN}

Desde la segunda mitad del siglo XX se ha producido un progresivo aumento en el número de alumnas que cursan estudios universitarios de modo que desde el último lustro de dicho siglo éstas superan ya en número a sus compañeros varones (Del Amo, 2009).

Esta situación actual, en la que se puede hablar de una mayor educación y formación de las mujeres (aunque esta no suponga realmente la igualdad de oportunidades) es fruto de un largo camino (Ballarín, 2004) que se inicia a mediados del siglo XIX cuando se estableció la obligatoriedad de la primera enseñanza para todos los españoles.

Desde un primer momento se establecieron diferencias en la formación de niños y niñas que tuvieron su reflejo en la formación de maestros y maestras. En particular se asumió, como veremos, que las maestras debían dedicarse a la educación de las niñas y los maestros a la de los niños. En consecuencia, las recientemente creadas Escuelas Normales (el Seminario Central de Maestros del Reino se abrió en Madrid en 1839 y la Escuela Central de Maestras en 1858) proporcionaban una formación diferente a los futuros maestros y maestras en función de su género.

Desde sus inicios el carácter de la enseñanza proporcionada en las escuelas normales es fuertemente culturalista y presta escasa atención a los conocimientos pedagógicos (Escolano, 1996); y ello pese a que Pablo Montesino, inspirador de la creación de las escuelas normales, propugna que lo esencial en la formación de los maestros tiene que ver con la moralidad y el carácter por encima de los meros conocimientos (Ávila, 2007).

Esta contradicción interna supone sólo en primero de los obstáculos a los que se enfrentaba el magisterio. A ello hay que unir las deficiencias en personal, dotación económica y recursos materiales y didácticos de las escuelas normales; que eran incluso más acusadas en el caso de las normales de maestras (Vega, 1986). Además, el maestro está extremadamente mal pagado en un país que dedica en torno a 17 veces más cantidad de dinero al ejército que a la enseñanza. Las maestras, hasta la nivelación de 1883, reciben ingresos incluso menores que sus colegas masculinos (Ávila, 2007).

En este contexto de dificultades, Ávila (2007) describe la formación de las maestras en una palabra: "discriminación". En particular, pone de manifiesto la ausencia de asignaturas científicas en los planes de estudio de maestras así como el ilustrativo hecho de que la Escuela Central de Maestras estuviera bajo la vigilancia y control de la Junta de Damas de Honor y Mérito; dando así mayor importancia en la formación de maestras a aspectos tales como la moral, la obediencia o el pudor.

En el presente trabajo se persigue analizar con detalle esta situación de discriminación. En particular se abordan dos objetivos, que se corresponden con dos distintos grados de concreción. En primer lugar, pretendemos describir las diferencias existentes a nivel global entre la formación de maestros y de maestras, especialmente en cuanto a las materias cursadas por cada uno de ellos. En segundo lugar, ponemos el foco en el caso concreto de la Geometría y abordamos el estudio de las diferencias y similitudes en la formación geométrica proporcionada a ambos colectivos. 


\section{Metodología}

Para lograr los objetivos anteriormente citados, abordamos una investigación de tipo histórico, entendida como un "proceso sistemático de búsqueda de información para responder preguntas sobre un fenómeno pasado con el objetivo de alcanzar una mejor comprensión acerca de instituciones, prácticas, tendencias y aspectos relacionados con la educación" (Gall, Borg y Gall, 1996: 514). Este tipo de estudios está recibiendo en tiempos recientes especial atención dentro de la Educación Matemática (González, 2009).

El estudio se realiza a partir de la información recabada de dos tipos de fuentes esencialmente:

1. Legislación de la época.

2. Libros de texto dedicados a la preparación de opositores.

El uso de estos dos tipos de fuentes se corresponde con los dos objetivos principales del trabajo. Por un lado, el estudio de la legislación nos proporciona información relevante sobre las diferencias existentes en los planes de estudios de los maestros y maestras elementales a nivel global. Sin embargo, a la hora de abordar el tratamiento dado a la Geometría en ambos casos, es necesario disponer de programas detallados de cada una de ellas, algo que no se establecía legalmente en la época. No obstante, como apunta Schubring (1987: 41) "la práctica docente no está tan determinada por los decretos ministeriales como lo está por los libros utilizados para la enseñanza". Además, Maz y Rico (2015: 54) ponen de manifiesto que "el análisis de textos escolares proporciona información sobre los contenidos, los conocimientos tratados y también sobre aspectos pedagógicos, curriculares o sociales". En el mismo contexto histórico que nos ocupa, Vega (1985) muestra la utilidad del estudio de libros de texto como fuente para la historia de la educación con un estudio sobre las corrientes pedagógicas imperantes en las normales españolas del XIX. En consecuencia, está justificado el análisis de manuales específicamente dirigidos a aspirantes a Maestros y Maestras de Primera enseñanza elemental.

Para abordar nuestro estudio seguimos a Picado y Rico (2011), quienes consideran tres focos para el análisis de manuales: autor, estructura del texto y contenido. En lo que se refiere al contenido distinguimos entre contenido matemático y contenido didáctico. Para el análisis del contenido matemático seguiremos las tres categorías propuestas por (Gómez, 2002): conceptos, sistemas de representación y fenomenología. El análisis de los planteamientos didácticos seguirá algunos de los indicadores propuestos por Maz y Rico (2015): rigor y precisión, interés social de las matemáticas, principios didácticos y principios filosóficos.

\section{ANÁLISIS DE LA LEGISLACIÓN}

\section{Visión general}

En la Ley de Instrucción Pública de 9 de septiembre de 1857 (generalmente denominada Ley Moyano, en honor de su impulsor) se estableció la obligatoriedad de la primera enseñanza para todos los españoles. Esta primera enseñanza, dividida a 
su vez en elemental y superior ya establecía diferencias en las materias cursadas por niños y niñas. En la Tabla 1 se muestran las diferencias existentes en ambos niveles, tal y como se recoge en el Título I de la Sección primera de la citada ley.

Tabla 1. Diferencias entre géneros en la Primera enseñanza

\begin{tabular}{cc}
\hline \multicolumn{2}{c}{ Primera Enseñanza Elemental } \\
\hline Niños & Niñas \\
\hline $\begin{array}{c}\text { Breves nociones de Agricultura, Industria y } \\
\text { Comercio, según las localidades. }\end{array}$ & Labores propias del sexo. \\
\hline \multicolumn{1}{c}{ Primera Enseñanza Superior } \\
\hline Niños & Niñas \\
\hline $\begin{array}{c}\text { Principios de Geometría, de Dibujo lineal y } \\
\text { de Agrimensura. }\end{array}$ & Elementos de Dibujo aplicado a las mismas \\
\hline $\begin{array}{c}\text { Nociones generales de Física y de Historia } \\
\text { natural acomodadas a las necesidades más } \\
\text { comunes de la vida. }\end{array}$ & Ligeras nociones de Higiene doméstica. \\
\hline
\end{tabular}

Además de las diferencias entre enseñanzas que Ilamaríamos prácticas, se observa cómo se hurtaba a las niñas el aprendizaje de la Geometría y de las Ciencias Físicas y Naturales. Se mantenían comunes la Doctrina cristiana e Historia sagrada, la Lectura y Escritura, la Gramática y Ortografía, la Aritmética y la Historia y Geografía.

En paralelo con estas diferencias, existían también una clara diferenciación en los estudios necesarios para convertirse en Maestro o Maestra de primera enseñanza (de niños o niñas, respectivamente). La Tabla 2 recoge en paralelo el articulado original de la Ley Moyano a este respecto y que se encuentra en al Capítulo III del Título III de la Sección tercera (dedicado a las enseñanza profesionales).

Como se observa, en el caso de la Primera enseñanza elemental, los requisitos son similares en ambos casos, en cuanto a que las materias exigidas son las mismas que se imparten en las escuelas elementales junto con algunos conocimientos pedagógicos. Así, pues, en el caso del acceso al cuerpo de Maestros de Primera enseñanza elemental, las diferencias reflejan exactamente las que se daban en la propia enseñanza elemental. Sin embargo la situación es ligeramente diferente en el caso de la Primera enseñanza superior. En este caso, a las maestras se les sigue exigiendo únicamente el conocimiento de las materias que van a impartir en sus escuelas, mientras que a sus compañeros varones se les pide también la adquisición de nociones de Álgebra; una materia que no van a tener que enseñar en su ejercicio profesional. Se continuaba así ensanchando la brecha en la formación científica.

Las diferencias anteriores imposibilitaban de facto el acceso de mujeres al cuerpo de Profesores de Escuela Normal; es decir, las imposibilitaba para formar nuevos docentes. De hecho, aunque la ley no señala esto explícitamente, es muy elocuente en el lenguaje empleado. Mientras se habla de Maestros y Maestras de Primera enseñanza, ya sólo se mencionan Profesores de Escuela Normal (art. 70, Cap. III, Tít. III, Sec. $\left.1^{\mathrm{a}}\right)$. 
Tabla 2. Diferencias entre géneros para convertirse en Maestro de Primera enseñanza

Primera Enseñanza

Maestro de niños

Artículo 68. Los estudios necesarios para obtener el título de Maestro de primera enseñanza elemental son: Catecismo explicado de la Doctrina cristiana. Elementos de Historia sagrada. Lectura. Caligrafía. Gramática castellana con ejercicios prácticos de composición. Aritmética. Nociones de Geometría, Dibujo lineal y Agrimensura. Elementos de Geografía. Compendio de la Historia de España. Nociones de Agricultura. Principios de educación y Métodos de enseñanza. Práctica de la enseñanza.

Artículo 69. Para ser Maestro de primera enseñanza superior, se requiere:

Primero. Haber estudiado las materias expresadas en el artículo anterior.

Segundo. Haber adquirido nociones de Álgebra, de Historia universal y de los fenómenos comunes de la Naturaleza.

No obstante, la redacción del artículo 71 se modificó (Ferrer, 1893: 26-27) primero en la Real Orden de 14 de marzo de 1877 y después en la Real Orden de 8 de junio de 1881 para ampliar y detallar los estudios conducentes al título de Maestra. Ese desarrollo detallado ya se había llevado a cabo en el caso de los Maestros con el Real Decreto de 20 de septiembre de 1858 (Ferrer, 1893: 25-26). En la Tabla 3 se recoge el listado de materias para cada modalidad.

Nuevamente se observan diferencias en las enseñanzas prácticas, donde la agricultura, la industria o el comercio se convierten en labores, higiene o música. Además, se mantienen las diferencias en cuanto a formación científica, no recibiendo las Maestras formación en Física, Ciencias Naturales o Álgebra y siendo más escasa la formación geométrica. Por otro lado, si se analiza con mayor detalle el plan de estudios observando el número de horas dedicadas a cada materia, también surgen diferencias interesantes. Por ejemplo, mientras los aspirantes a Maestro elemental recibían lecciones diarias de Lectura durante dos cursos, las aspirantes a Maestra elemental recibían lecciones alternas un curso y tres lecciones semanales el segundo. Esta práctica liberaba tiempo para asignaturas como Labores de punto y costura que eran de lección diaria. La formación pedagógica era similar, 2 lecciones por semana durante un curso tanto en el nivel elemental como en el superior, pero las Maestras debían cursar Práctica de la Enseñanza en ambos niveles (que no aparece con asignación temporal en el texto legal). 
Tabla 3. Planes de estudios de los futuros maestros y maestras

\begin{tabular}{|c|c|}
\hline \multicolumn{2}{|c|}{ Maestro/a de Primera Enseñanza Elemental } \\
\hline Maestro de niños & Maestra de niñas \\
\hline $\begin{array}{c}\text { Doctrina cristiana y nociones de Historia sagrada (2 } \\
\text { cursos). } \\
\text { Teoría y Práctica de la Lectura (2 cursos). } \\
\text { Teoría y Práctica de la Escritura (2 cursos). } \\
\text { Lengua castellana con ejercicios de análisis, } \\
\text { composición y ortografía (2 cursos). } \\
\text { Aritmética (1 curso). } \\
\text { Nociones de Geometría, Dibujo lineal y Agrimensura } \\
\text { (1 curso). } \\
\text { Elementos de Geografía y nociones de Historia de } \\
\text { España (1 curso). } \\
\text { Nociones de Agricultura (1 curso). } \\
\text { Principios de Educación y Métodos de enseñanza (1 } \\
\text { curso). }\end{array}$ & $\begin{array}{c}\text { Primer curso: } \\
\text { Explicación del catecismo de la Doctrina cristiana. } \\
\text { Práctica de la Lectura. } \\
\text { Práctica de la Escritura. } \\
\text { Elementos de Gramática Castellana. } \\
\text { Elementos de Aritmética. } \\
\text { Labores de punto y costura. } \\
\text { Nociones de Geografía y particularmente la de España. } \\
\text { Dibujo aplicado a las labores con ligeras nociones de } \\
\text { Geometría. } \\
\text { Principios de canto y solfeo. } \\
\text { Práctica de la enseñanza. } \\
\text { Nociones de Historia sagrada. } \\
\text { Teoría y Práctica de la Lectura. } \\
\text { Tegundo curso: } \\
\text { prácticos de ortografía. } \\
\text { Continuación de la Gramática y análisis razonado de } \\
\text { ejercicios de composición. } \\
\text { Continuación de la Aritmética hasta las proporciones y } \\
\text { ejercicios de resolución de problemas. } \\
\text { Principios de Educación, métodos de enseñanza y } \\
\text { organización de escuelas. } \\
\text { Nociones de Historia de España. } \\
\text { Continuación de las labores. } \\
\text { Continuación de Dibujo. } \\
\text { Continuación de Música. } \\
\text { Práctica de la enseñanza. } \\
\end{array}$ \\
\hline \multicolumn{2}{|c|}{ Maestro/a de Primera Enseñanza Superior } \\
\hline Maestro de niños & Maestra de niñas \\
\hline $\begin{array}{c}\text { Doctrina cristiana explicada e Historia sagrada. } \\
\text { Lengua castellana con ejercicios de análisis, } \\
\text { composición y ortografía. } \\
\text { Teoría y Práctica de la Lectura. } \\
\text { Teoría y Práctica de la Escritura. } \\
\text { Complemento de la Aritmética y nociones de Álgebra. } \\
\text { Elementos de Geometría, Dibujo lineal y Agrimensura. } \\
\text { Elementos de Geografía e Historia. } \\
\text { Conocimientos comunes de Ciencias Físicas y } \\
\text { Naturales. } \\
\text { Práctica de Agricultura. } \\
\text { Nociones de Industria y Comercio. } \\
\text { Pedagogía. }\end{array}$ & $\begin{array}{c}\text { Ampliación de las lecciones de Doctrina cristiana e } \\
\text { Historia sagrada. } \\
\text { Lectura expresiva y cultivo de la inteligencia por este } \\
\text { medio. } \\
\text { Ejercicios caligráficos y redacción de documentos más } \\
\text { usuales. } \\
\text { Ampliación de la Gramática con ejercicios de análisis } \\
\text { lógico. } \\
\text { Ampliación de la Aritmética comprendiendo las } \\
\text { proporciones y aplicación de esta Teoría. } \\
\text { Nociones de Higiene y Economía doméstica. } \\
\text { Ampliación de la Pedagogía. } \\
\text { Labores de primor y de adorno. } \\
\text { Dibujo de adorno y figura. } \\
\text { Práctica de la enseñanza. }\end{array}$ \\
\hline
\end{tabular}


Maestro de Escuela Normal

\begin{tabular}{cc}
\hline \multicolumn{1}{c}{ Maestro de Escuela Normal } \\
\hline Hombres & Mujeres \\
\hline Retórica y Poética. & No contemplado en la legislación \\
Pedagogía. & \\
Noticia de las disposiciones oficiales relativas a \\
Primera Enseñanza. \\
Religión y Moral.
\end{tabular}

\section{La Geometría}

Por lo que respecta a la Geometría, la primera diferencia salta a la vista sin más que observar el nombre de las respectivas materias: Nociones de Geometría, Dibujo lineal y Agrimensura en el caso de los maestros y Dibujo aplicado a las labores con ligeras nociones de Geometría en el de las maestras. Parece claro que la Geometría constituye el contenido central de la asignatura masculina y sólo una parte de la femenina. Los maestros reciben nociones y las maestras únicamente ligeras nociones. Ambas materias, sin embargo, tenían la misma carga horaria de tres lecciones semanales durante un curso (Ferrer, 1893: 25, 27).

La distinta carga matemática de cada una de ambas materias se pone igualmente de manifiesto al observar que en el caso de las maestras se cursaba en el primer año, simultáneamente con los Elementos de Aritmética, mientras que los maestros tenían la condición de que "la Aritmética preceda a las Nociones de Geometría, Dibujo lineal y Agrimensura" (Ferrer, 1893: 25). Es de suponer, por tanto, una mayor carga conceptual en la signatura masculina dado que se imponían unos prerrequisitos matemáticos mayores.

\section{Los manuales analizados}

A finales del XIX se publicó una colección de textos titulada Guía de opositores a Escuelas elementales y del grado superior. El director de la colección fue Manuel Rodríguez Navas y, según se indicaba en la página final de cada libro, estaba compuesta por:

Obras escritas con sujeción a los programas para oposiciones a las Escuelas elementales y de párvulos dotadas con 825 pesetas, y de grado superior, dotadas con 2000 pesetas, o más, publicados por el Ministerio de Fomento con fechas 10 de octubre y 12 de Noviembre de 1894, y aprobados por el Consejo de Instrucción Pública.

Así pues, estos textos son una interesante fuente de información a la hora de analizar los contenidos que se exigían en las oposiciones a los futuros maestros y que seguramente reflejarían con cierta fidelidad los contenidos de los planes de estudio. Además, en cuanto a las diferencias que nos interesa observar, las obras publicadas en esta colección resulta útiles puesto que, según leemos:

En estas obras va indicado con tipos diferentes, y con claridad, lo que pertenece a cada programa elemental o superior, y lo que es particular para Profesores o Profesoras. 
En esta colección de 14 tomos se encuentran manuales consagrados todas las materias que componían los planes de estudios, desde Historia sagrada, hasta Pedagogía. Con algunas de ellas comparten incluso el título. Vamos a fijarnos en las dos obras dedicadas a la geometría:

- Geometría y Agrimensura (Pitaluga, 1895).

- Nociones de Geometría con aplicaciones a las labores y corte de prendas (Barceló y Luis, 1895).

\section{La Geometría y Agrimensura de Vicente Pitaluga}

La Geometría y Agrimensura fue escrita por Vicente Pitaluga y García, doctor graduado en Ciencias exactas, profesor numerario de Aritmética y Geometría en "El Fomento de las Artes" y socio de mérito a perpetuidad en este centro por servicios prestados en la enseñanza. También fue autor del texto Aritmética y Nociones de Álgebra (Pitaluga, 1896) publicado en esta misma colección.

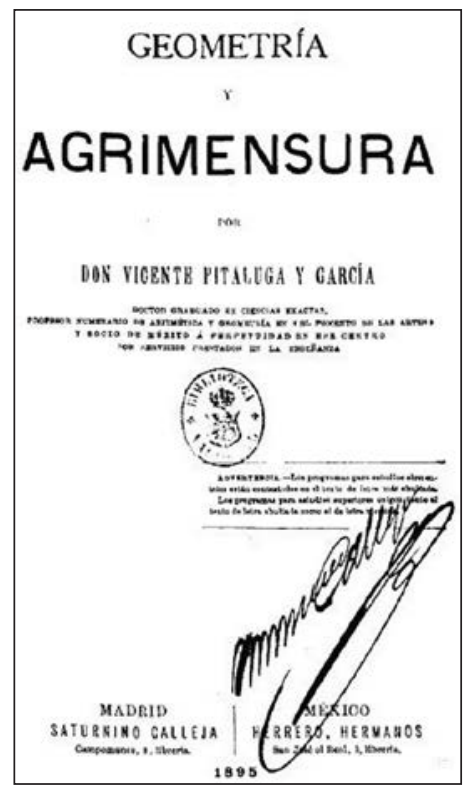

Figura 1. Portada de la Geometría y Agrimensura de Vicente Pitaluga

El texto se desarrolla a lo largo de setenta y nueve páginas y contiene los programas de Geometría y Agrimensura correspondientes al grado elemental (treinta y una lecciones) y superior (cuarenta y dos lecciones) para las escuelas de niños. Los contenidos de ambos programas se desarrollan simultáneamente en tópicos que incluyen las lecciones del grado elemental y del grado superior. Los contenidos de cada lección que son específicos del grado superior aparecen con una tipografía más reducida. En la tabla siguiente se reflejan esquemáticamente los contenidos de la obra. 
Tabla 4. Contenidos de la Geometría y Agrimensura de Vicente Pitaluga

\begin{tabular}{|c|c|c|}
\hline \multirow[b]{2}{*}{ Tópicos } & \multicolumn{2}{|c|}{ Lecciones del } \\
\hline & grado elemental & grado superior \\
\hline Definiciones preliminares & 1 & 1 y 2 \\
\hline Ángulos & 2 & 3 \\
\hline Líneas perpendiculares y oblicuas & 3 & 5 \\
\hline Líneas paralelas & 4 & 4 y 6 \\
\hline Polígonos & 5 & 7 \\
\hline Igualdad y semejanza de triángulos & 6 & 8 \\
\hline Cuadriláteros y paralelogramos & 7 & 9 \\
\hline Círculo y circunferencia & 8 & 10,11 y 12 \\
\hline Medida de la circunferencia y de los ángulos & 9 & 13,14 y 15 \\
\hline Líneas proporcionales & 10 & 16 y 17 \\
\hline Casos de proporcionalidad & 11 & 19 \\
\hline Polígonos inscritos y circunscritos & 12 & 18 \\
\hline Modo de inscribir polígonos & 13 & 21 \\
\hline Polígonos semejantes & 14 & 18 y 20 \\
\hline Áreas & 15 & 22 \\
\hline $\begin{array}{l}\text { Área de polígonos regulares, sectores de círculo, } \\
\text { elipse, línea espiral y curvas mecánicas }\end{array}$ & 16 & $23,24,25$ y 26 \\
\hline Plano: sus distintas posiciones & 17 & 27 \\
\hline Ángulos diedros y poliedros & 18 & 28 \\
\hline Prismas & 19 & 29 \\
\hline Pirámides & 20 & 30 \\
\hline Poliedros regulares & 21 & 31 \\
\hline Cuerpos redondos & 22 & 33 \\
\hline Conos & 23 & 32 \\
\hline Esfera. Volúmenes & 24 & $34,35,36$ y 37 \\
\hline Elipse & 25 & 25 y 26 \\
\hline Ideas generales (Agrimensura) & 26 & 38 \\
\hline Escalas & 27 & \\
\hline Alineaciones & 28 & 39 y 40 \\
\hline Casos especiales de alineación & 29 & \\
\hline Medición de alturas & 30 & 41 \\
\hline Levantamiento de planos & 31 & 42 \\
\hline
\end{tabular}

\section{Las Nociones de Geometría con aplicaciones a las labores y corte de prendas de Juan Barceló y Vicenta de Luis}

Nociones de Geometría con aplicaciones a las labores y corte de prendas fueron escritas por Juan Barceló y García, maestro superior y profesor del Colegio Politécnico Matritense, y por Vicenta de Luis y Gil, maestra superior y directora de un colegio de Madrid. Es interesante señalar que, según el listado de obras que formaban la colección que aparece en la página final de las mismas, este texto debía ser escrito por Vicente Pitaluga. Sin embargo, los autores fueron finalmente otros. La obra ocupa un 
total de ciento quince páginas y contiene los programas de Nociones de Geometría correspondientes al grado elemental (veinte lecciones) para las escuelas de niñas y párvulos y al grado superior (treinta y tres lecciones) para las escuelas de niñas.

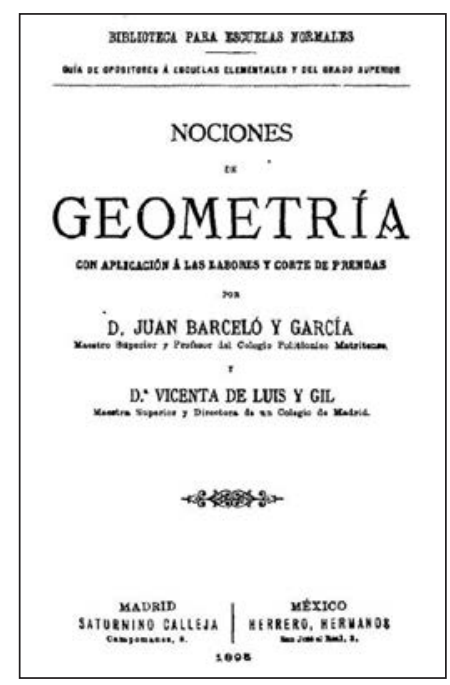

Figura 2. Portada de las Nociones de Geometría de Juan Barceló y Vicenta de Luis.

Los contenidos de ambos programas se desarrollan simultáneamente en tópicos que incluyen las lecciones del grado superior y del grado elemental. Nuevamente los contenidos específicos del grado superior aparecen con una tipografía reducida. En la tabla siguiente se reflejan esquemáticamente los contenidos de la obra.

Tabla 5. Contenidos de las Nociones de Geometría de Juan Barceló y Vicenta de Luis

\begin{tabular}{|c|c|c|}
\hline \multirow[b]{2}{*}{ Tópicos } & \multicolumn{2}{|c|}{ Lecciones del } \\
\hline & grado elemental & grado superior \\
\hline Geometría y sus aplicaciones & 1 & 1 \\
\hline Nociones generales & 2 & 2 \\
\hline Formas geométricas & & 3 \\
\hline Ángulos & 3 & 4 \\
\hline Posiciones y división de la línea recta & 6 & 5 \\
\hline Clases y medidas de ángulos & 5 & 6 \\
\hline Líneas perpendiculares y oblicuas & 6 & 7 \\
\hline Clases de triángulos & 10 y 11 & 8 \\
\hline Construcción de triángulos & & 9 \\
\hline Polígonos & 10 y 13 & 10 \\
\hline Cuadrilátero & 12 & 11 \\
\hline
\end{tabular}




\begin{tabular}{|c|c|c|}
\hline Igualdad y semejanza de los cuadriláteros. Áreas & 14 & 12 \\
\hline $\begin{array}{l}\text { Triangulación de los polígonos regulares. Áreas de los } \\
\text { polígonos. }\end{array}$ & 14 & 13 \\
\hline $\begin{array}{l}\text { División de rectas en partes iguales y proporcionales y } \\
\text { construcción se polígonos semejantes }\end{array}$ & 7 y 8 & 14 \\
\hline Circunferencia y círculo & 4 y 5 & 15 \\
\hline $\begin{array}{l}\text { Razón de la circunferencia al diámetro. Problemas } \\
\text { relativos a la circunferencia. }\end{array}$ & 15,16 y 17 & 16 \\
\hline Planos en sus diferentes posiciones. Ángulos diedros & 18 & 17 \\
\hline Poliedros & 18 & 18 \\
\hline Pirámide & 19 & 19 \\
\hline Pirámide truncada & & 20 \\
\hline Prismas. Paralelepípedo. Cubo & 19 & 21 \\
\hline Cono, cilindro, esfera & 20 & 22 \\
\hline Cilindro, esfera, centro, radio, diámetro, eje, polos & & 23 \\
\hline Volúmenes & & 24 \\
\hline Combinaciones de líneas rectas & & 25 \\
\hline Combinaciones de líneas curvas & & 26 \\
\hline Aplicaciones de la geometría al corte de patrones & & 27 \\
\hline Corte de patrones & & 28 \\
\hline Adaptación de patrones & & 29 \\
\hline Corte de chaqueta & & 30 \\
\hline Diferentes conformaciones del busto & & 31 \\
\hline $\begin{array}{l}\text { Enseñanza del corte y la confección en las escuelas } \\
\text { de niñas }\end{array}$ & & 32 \\
\hline Enseñanza de la Geometría en las escuelas de niñas & & 33 \\
\hline
\end{tabular}

\section{ANÁLISIS COMPARADO DEL CONTENIDO DE LOS MANUALES}

\section{Contenido matemático}

\section{Conceptos}

Los objetos matemáticos presentados en ambos manuales son idénticos. Sin embargo, las propiedades presentadas y la profundidad en el tratamiento varían. Por ejemplo, mientras en el texto de maestros se justifica el hecho de que sólo existen 5 poliedros regulares, en el texto destinado a las maestras tan sólo se definen y describen. Las diferencias entre ambos textos aparecen al observar algunas de las construcciones que se presentan en ellos. En el caso del texto de Barceló y de Luis se muestran construcciones que van a ser aplicadas en la parte de la obra dedicada al patronaje. Por ejemplo, se muestra un método para construir un polígono semejante a uno dado (Barceló y Luis, 1895: 66):

el método preferible es unir todos los vértices del polígono por medio de rectas con un punto cualquiera exterior a él, tomar en ellas una longitud igual a la semejanza [...] y trazar paralelas respectivamente a los lados del polígono. 


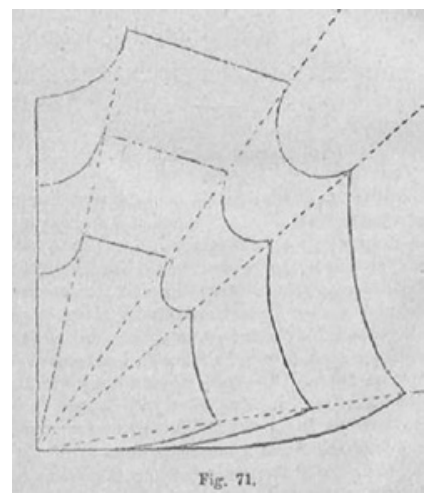

Figura 3. Aumento y disminución de una prenda (Barceló y Luis, 1895, p. 110)

Este método es posteriormente utilizado para presentar un procedimiento para aumentar o disminuir una prenda (Figura 3). El manual para maestros no presenta esta construcción.

En el caso del manual de Pitaluga, se presentan construcciones con una menor aplicación práctica, pero con mayor importancia matemática. Encontramos, por ejemplo, el procedimiento para dividir un segmento en media y extrema razón (Figura 4). La importancia de esta construcción radica en "su aplicación en algunos problemas de inscripción de polígonos" (Pitaluga, 1895: 36).

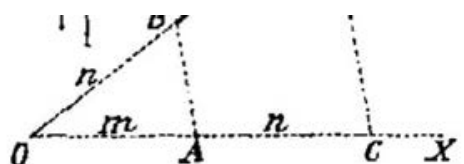

Fig. 28.
Ses $\ddot{A} B(\hat{f i g} .29)$ la rects que se trate de dividir en media $y$ extrems razón. Levántese en uno de sus extremos, $B$, una perpendicular y tómese sobre ella una magni-

tud $B O=\frac{1}{2} A B ;$ describase tesule $O$, como centro, y con $O B$ por radio, una cireunferencis; trácese $A N O M$ y llévese $A N$ gobre $A B$ : el punto $C$ dividirá á la recta $A B$ en media y extrema rnzón.

En efecto; los triángnlos $A B M$ y $A B N$, que tienen el ángulo $A$ común, y el $B M A=A B N$, nos den:

$$
\frac{A M}{A B}=\frac{A B}{A N}
$$

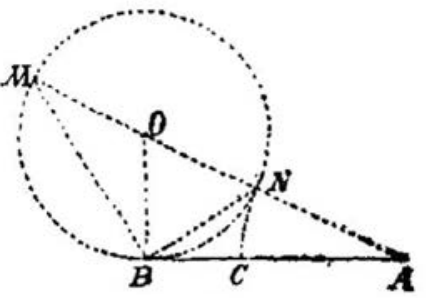

Fig. 29.

De donde

$$
\frac{A B-A B}{A B}=\frac{A B \cdot A V}{A \cdot V}
$$

$\hat{O}$ sea

$$
\frac{A N}{A B}=\frac{B C}{A N}
$$

Figura 4. División de un segmento en media y extrema razón (Pitaluga, 1895, p. 36) 


\section{Sistemas de representación}

El modo en que ambos textos denotan los objetos geométricos es idéntico y es similar al de los manuales de la época. Los puntos se representan con letras mayúsculas y los segmentos bien con letras minúsculas, bien señalando sus extremos. Los polígonos se denotan enumerando sus vértices. En las escasas ocasiones en las que intervienen longitudes desconocidas, éstas se denotan con letras minúsculas.

En las construcciones y figuras planas, los datos aparecen siempre en trazo continuo, mientras que las líneas auxiliares aparecen el trazo discontinuo.

Sólo en la obra de Pitaluga dedicada a maestros aparecen los desarrollos planos de los poliedros regulares y no sólo su representación tridimensional (Figura 5).

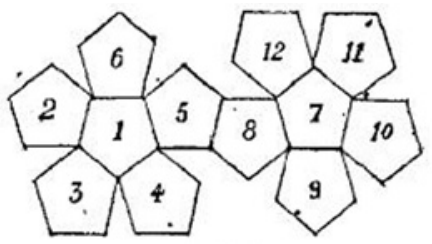

Fig. 52.

Figura 5. Desarrollo plano del dodecaedro (Pitaluga, 1895, p. 61)

Al trabajar con razones y proporciones, Pitaluga utiliza siempre notación fraccionaria para las razones y el símbolo de igualdad para indicar la igualdad de dos razones en una proporción. Por su parte, Barceló y de Luis alternan esta práctica con la notación clásica, más arcaica (Figura 6).

$$
C: C^{\prime}:: R: R^{\prime} . \frac{E D}{E C}=\frac{E A}{E A} .
$$

Figura 6. Distintas notaciones para razones y proporciones (Barceló y Luis, 1895, pp. 73, 75)

\section{Fenomenología}

En ambos manuales se presenta la geometría como una ciencia que trata sobre entes abstractos. Esto es especialmente claro en el caso de Pitaluga (1895: 11), quien define la Geometría como "la ciencia que trata de la extensión" cuyo objeto es "el estudio de las propiedades de las figuras o extensiones determinadas". Barceló y Luis (1895: 9) sí parecen relacionar esta ciencia con la realidad en cierto modo ya que definen la Geometría como "una ciencia que tiene por objeto el estudio del espacio limitado que ocupan los cuerpos en la naturaleza".

En cualquier caso, ninguno de los dos textos presenta los objetos geométricos como abstracciones de objetos reales, ni motiva las definiciones o propiedades con problemas reales. Por el contrario, las aplicaciones aparecen a posteriori, una vez que se ha presentado todo el material teórico. 
Estas aplicaciones, como ya se ha mencionado anteriormente, son claramente distintas según los destinatarios de la obra. Mientras en el caso de los maestros se indica que "una de las más importantes [aplicaciones de la Geometría es] la que da reglas para la medición de terrenos, o sea la Agrimensura" (Pitaluga, 1895: 11), las maestras aplicaban sus conocimientos geométricos al corte de patrones ya que "todas las prendas de vestir [...] después de cortadas tienen figuras geométricas" (Barceló y Luis, 1895: 102).

Así pues, las aplicaciones prácticas que se tratan en el manual para maestros son: el trazado de escalas, la medición de rectas y ángulos sobre el terreno, el trazado de alineaciones, la medición de alturas accesibles e inaccesibles y el levantamiento de planos.

En el caso de las maestras, las aplicaciones son más escasas: uso y trazado de escalas y ampliación o reducción de patrones.

\section{Planteamientos didácticos}

Rigor y precisión

Ambos textos son prácticamente idénticos en lo que respecta al rigor y precisión en el lenguaje utilizado y en el tratamiento dado a la materia.

El lenguaje utilizado en su redacción es claro y conciso, como puede apreciarse en el texto siguiente relativo a las definiciones de polígono y diagonal.

Polígonos: su clasificación.-Llámase polígono la superficie limitada por rectas; éstas se llaman lados del polígono, y sns intersecciones, vírtices. Diagonal de un poligono es la recta que nne dos vértices no consecutivos. Perimetro de un poligono es ln suma de sus lados.

Polígono cóncavo es el que puede ser cortado por nna recta en más de dos puntos; convexo el que sólo pucde ser cortado en dos puntos por nua recta.

Polígono equilátero es nquel que tiene todos sus lados ignales; equiángulo, el que tiene iguales todos sus ángulos, $\mathrm{y}$ regular, el que es simultáneamente eqnilátero y equíingulo.

Figura 7. Algunas definiciones (Pitaluga, 1895, p. 22)

Los dos manuales contienen figuras intercaladas en el texto. Esta costumbre no era común en todos los libros de Geometría contemporáneos dedicados a la enseñanza, en muchos de ellos las figuras aparecían al final en láminas desplegables.

En ambos casos encontramos demostraciones de algunas proposiciones elementales. Las demostraciones siempre están indicadas únicamente para los aspirantes a maestro o maestra de grado superior (aparecen con tipografía reducida). En general no coinciden las demostraciones que se dan en ambos textos. Por ejemplo, Pitaluga (1895: 24) se conforma con señalar que para probar que los ángulos de un triángulo suman 
dos rectos "bastaría con trazar por cualquiera de los vértices una paralela al lado puesto" mientras que Barceló y Luis (1895: 37) detallan todos los pasos de la demostración.

En ninguna de las dos obras se proponen ejercicios o problemas para que los opositores pongan a prueba sus conocimientos.

\section{Interés social de las matemáticas}

El texto de Pitaluga es puramente matemático. La única indicación que se hace a la importancia o interés de la Geometría la encontramos al inicio de la obra donde se dice que "la importancia de la Geometría se reconoce por sus innumerables aplicaciones" (Pitaluga, 1895: 11). Ni siquiera cuando, en la parte final de la obra se presentan algunas de esas aplicaciones se dan ejemplos prácticos en los que puedan resultar de interés. Por ejemplo, en la lección XXIX del programa elemental se muestra cómo trazar una alineación recta en presencia de un obstáculo, pero no se comentan situaciones concretas en las que esto pueda ser útil o necesario.

El texto de Barceló y de Luis es más interesante a este respecto y se detiene más en justificar el interés de la enseñanza de Geometría. Además, este interés va más allá de las meras aplicaciones prácticas, y se centra en la capacidad formativa de esta materia. Así, los autores señalan que "la Geometría proporciona a los maestros ocasiones frecuentes de instruir deleitando [...] y de favorecer el desarrollo intelectual de sus educandos" (Barceló y Luis, 1895: 10).

\section{Principios didácticos}

En el texto dedicado a los maestros no existe ningún tipo de consideración ni de recomendación didáctica respecto a la enseñanza o el aprendizaje de la Geometría. Como ya hemos mencionado, es un texto que se limita a presentar contenidos matemáticos.

El manual cuyas destinatarias son las futuras maestras, por el contrario, sí que presenta comentarios al respecto. Posiblemente esto se deba a que los autores de este texto era maestros En la lección inicial, por ejemplo, se dan propuestas sobre el uso de materiales manipulativos en el aula (Barceló y Luis, 1895: 10-11):

Un trozo de papel convenientemente doblado servirá para demostrar varias teorías referentes a líneas paralelas, [...], a triángulos. Por medio de varias pajaritas de papel puestas en una mesa o en el suelo de tal modo que por el orden de colocación formen varias líneas iguales, de esta manera [formando un retículo] los alumnos podrán comprender perfectamente de un modo práctico muchas reglas y aun resolver muchos problemas de Aritmética

Además, la última lección del programa superior (XXXIII), aunque breve, se titula "Enseñanza de la Geometría en las escuelas de niñas". En esta lección se dan indicaciones metodológicas respecto al orden y la profundidad de los contenidos que se deben presentar a las niñas. Por ejemplo, se indica que la enseñanza debe hacerse "prescindiendo de los teoremas y las demostraciones de estos mismos pues hay que suprimir todo lo que pueda resultar árido y pesado [...] y que además no tenga ninguna aplicación práctica" (Barceló y Luis, 1895: 115). 


\section{Principios filosóficos}

Nuevamente sólo aparecen comentarios que ilustran la posición filosófica de los autores en el texto dedicado a las futuras maestras. Aparecen intercaladas en los comentarios de carácter didáctico que acabamos de comentar y en ellas se describe con toda crudeza el papel de la mujer en la sociedad española de finales del XIX. Presentamos a continuación algunos fragmentos:

Para la mujer, este conocimiento [se refiere a la Geometría] es de necesidad en las labores y corte de prendas de vestir, a que debe dedicar desde niña una preferente atención si quiere disponerse a cumplir debidamente las altas funciones de madre de familia y ordenadora de su casa, a que la naturaleza la destina (Barceló y Luis, 1895: 11).

Es de grandísima importancia en los colegios [de niñas] la enseñanza del corte y confección de prendas de vestir, tanto como la escritura y la lectura, pues cualquiera que sea la posición de la mujer, debe estar práctica en esta clase de conocimientos. Si es rica, sabrá mandarlo, y también conocerá con más facilidad los defectos que tenga una prenda. Si fuese pobre, puede recurrir al trabajo de costura, como medio para atender a las necesidades de su vida (Barceló y Luis, 1895: 114).

Es muy importante esta enseñanza [la del corte y confección], porque la que hoy es niña, algún día será mujer y desempeñará un papel muy principal en la sociedad, pudiendo hacer grandes economías en los gastos de la familia, si sabe confeccionar ella misma las prendas de vestir (Barceló y Luis, 1895: 114).

Esta asignatura [se refiere a la Geometría] debe ser puramente práctica; cuanto en ella se enseñe debe tener aplicación directa a las labores y corte de prendas, que es lo que más interesa saber a la mujer; aunque no debe descuidarse la enseñanza de la Geometría con aplicación al dibujo, por lo que a la mujer se refiere, porque el dibujo es una de las artes bellas que con más éxito puede la mujer cultivar, ya como entretenimiento agradable y de exquisita delicadeza estética, ya como un medio honesto y decoroso de proporcionarse recursos para el sostenimiento propio y el de su familia (Barceló y Luis, 1895: 115).

\section{Discusión y CONCLUSIONES}

Hemos comenzado el trabajo poniendo de manifiesto las diferencias existentes en la primera enseñanza de niños y niñas a finales del siglo XIX en España. Esta diferencia se reflejaba claramente, a nivel de legislación, en la formación de los Maestros y Maestras encargados de proveer esa primera enseñanza. Como ya apuntaba Ávila (2007) se observa una menor carga de contenidos científicos en los planes de estudio de las maestras sustituidas por contenidos considerados "femeninos". En definitiva, queda claro que a finales del siglo XIX la formación de Maestros y Maestras estaba claramente diferenciada, no solamente debido a las diferencias de género en la propia Enseñanza elemental, sino por un claro reparto de roles en la sociedad y por la consideración de que las "mentes femeninas" no eran aptas ciertos conocimientos.

Al centrarnos en el caso de la Geometría y descender del nivel legislativo al del currículo detallado, tal y como se reflejaba en los manuales para opositores de la 
época, hemos observado que no existían grandes diferencias en cuanto al contenido matemático. A ese respecto, la formación puramente geométrica de los maestros y las maestras era totalmente equiparable. De hecho, se observa una mayor preocupación por los aspectos didácticos en el manual dedicado a las maestras, por lo que diríamos que la formación recibida por estas era más global y no estaba únicamente centrada en el contenido, sino que también ponía el foco en los métodos de enseñanza y en la utilidad e interés práctico y formativo de la disciplina.

Al centrarnos en la fenomenología o en las aplicaciones prácticas que se dan a dichos conocimientos ya comenzamos a vislumbrar el papel pasivo que se reservaba a la mujer en la época. Esta visión se completa al rastrear la posición ideológica de los autores (uno de ellos mujer). Se constata pues la labor ideológica que ejercían las escuelas normales y cómo éstas "proyectaban sobre los alumnos ciertos mensajes ideológicos" (Cerezo, 1986).

Es reseñable el hecho de que el texto dedicado a los maestros no sólo carece de indicaciones pedagógicas, sino que es ideológicamente neutro. Esto encaja con el carácter general de la enseñanza proporcionada en las escuelas normales, según señala Escolano (1996).

Se transmite así la idea de que la ciencia "pura" sólo está destinada a los hombres, mientras que si algunas mujeres acceden a ella es únicamente para actuar como docentes. En definitiva, como ya señala Escolano (1997: 15) el libro de texto "es un espejo de la sociedad".

\section{REFERENCIAS BIBLIOGRÁFICAS}

Ávila, A. (2007). La formación de los maestros en España: una deuda histórica. Historia de la educación: Revista interuniversitaria, 26, 327-340.

Ballarín, P. (2004). Género y políticas educativas. XXI Revista de Educación, 6, 35-42.

Barceló, J., y Luis, V. de (1895). Nociones de Geometría con aplicaciones a las labores y corte de prendas. Madrid: Saturnino Calleja.

Cerezo, J. F. (1986). Componentes ideológicos de la formación de maestros en CastillaLeón (1900-1936). Historia de la educación: Revista interuniversitaria, 5, 401-414.

Del Amo, M. C. (2009). La educación de las mujeres en España: de la "amiga" a la universidad. CEE Participación Educativa, 11, 8-22.

Escolano, A. (1997). Introducción. En A. Escolano (coord.), Historia ilustrada del libro escolar en España: del Antiguo Régimen a la Segunda República (pp. 13-17). Madrid: Pirámides.

Escolano, A. (1996). Memoria de la formación de maestros. Aula, 8, 311-327.

Ferrer, P. (1893). Tratado de la legislación de primera enseñanza vigente en España (séptima edición). Madrid: Librería de la viuda de Hernando y $C^{a}$.

Gómez, P. (2002). Análisis didáctico y diseño curricular en matemáticas. Revista EMA, 7(3), 251-292.

Maz, A., y Rico, L. (2015). Principios didácticos en textos españoles de matemáticas en los siglos XVIII y XIX. Revista Latinoamericana de Investigación en Matemática Educativa, 18(1), 49-76. 
Picado, M., y Rico, L. (2011). Análisis de contenido en textos históricos de matemáticas. PNA, 6(1), 11-27.

Pitaluga, V. (1895). Geometría y Agrimensura. Madrid: Saturnino Calleja.

Pitaluga, V. (1896). Aritmética y Nociones de Álgebra. Madrid: Saturnino Calleja.

Schubring, G. (1987). On the methodology of analysing historical textbooks: Lacroix as textbook author. For the Learning of Mathematics, 7(3), 41-51.

Vega, L. (1985). Las corrientes pedagógicas europeas a la luz del movimiento normalista español del siglo XIX. Historia de la educación: Revista interuniversitaria, 4, 119-138.

Vega, L. (1986). La eficacia interna de la formación de maestras en el siglo XIX. Historia de la educación: Revista interuniversitaria, 5, 235-250. 